\title{
Maßnahmen und Instrumente der städtischen Wärmeplanung
}

Für eine zielgerichtete und klimafreundliche Umgestaltung der städtischen Wärmeversorgung bedarf es eines umfangreichen Instrumentariums. Die Stadt Hamburg setzt bereits mehrere, aufeinander aufbauende Instrumente ein und entwickelt diese stets weiter, um der großen Herausforderung der Wärmewende gerecht zu werden. Von Lubow Hesse

D er Senat der Freien und Hansestadt Hamburg hat sich im Rahmen des Hamburger Klimaplans das Ziel gesetzt, die eigenen $\mathrm{CO}_{2}$-Emissionen um mindestens 80 Prozent gegenüber dem Jahr 1990 zu reduzieren. Bis 2030 sollen sie halbiert werden. Ein weiteres Ziel, der Ausstieg aus der Kohleverbrennung in der Fernwärme bis 2030, wurde erst kürzlich von der Hamburger Bürgerschaft gesetzlich verankert. Für einen Ballungsraum und Wirtschaftsstandort wie Hamburg ist der Weg besonders ambitioniert: Es steht wenig Fläche für die Nutzung Erneuerbarer Energien zur Verfügung, und es kommt infolge der Urbanisierung mit dem Anstieg des Energiebedarfs zu einer zunehmenden $\mathrm{CO}_{2}$-Belastung. Vor allem der Hamburger Wärmesektor ist für einen Großteil dieser Belastung verantwortlich - hier werden rund 50 Prozent der Endenergie für die Bereitstellung von Warmwasser, Raum- und Prozesswärme verbraucht (BUE 2018). Folglich gilt es gerade in diesem Sektor, den Handlungsraum weit zu öffnen und zügig Wege zur Steigerung der Gebäudeeffizienz sowie des Anteils Erneuerbarer Energien zu beschreiten. Aufgrund ökonomischer Restriktionen energetischer Gebäudesanierungsmaßnahmen und der begrenzt verfügbaren Ressourcen im urbanen Raum steht man stets vor der besonderen Herausforderung, eine volkswirtschaftlich sinnvolle und realistisch erreichbare Kombination beider Stellschrauben zu finden.

Ein bedeutender Weg für Hamburg ist der Ausbau der leitungsgebundenen Wärmeversorgung. Vor allem in Gebieten mit verdichteter Bebauung kann über Wärmenetze ein hohes Potenzial zur Dekarbonisierung der Wärmeversorgung gehoben werden. Im Vergleich zu Einzelversorgungslösungen auf Gebäudeebene lassen sich höhere Anteile erneuerbarer Energien erreichen und es kann gezielter Einfluss auf eine schnellere Umstellung der Brennstoffe im Zuge klimapolitischer Kurswechsel genommen werden. Ein weiterer Vorteil ist, dass sich Kombinationen mit Technologien zur Sektorenkopplung sinnvoller und wirtschaftlicher realisieren lassen. Mit dem beschlossenen Rückkauf des zentralen Fernwärmenetzes schafft sich die Stadt Hamburg eine entscheidende Einflussmöglichkeit, einen Großteil der Hamburger Wärmeinfrastruktur zukunftsfähig umzugestalten und den $\mathrm{CO}_{2}$-Ausstoß erheblich zu reduzieren.

In Anbetracht der Komplexität des Wärmesektors, insbesondere mit Blick auf die heterogenen Eigentums- sowie Versorgungsstrukturen und der unvorhersehbaren Dynamik der Entscheidungsprozesse von Investoren und Gebäudeeigentümer/innen, kann es keine gesamtstädtische Standardlösung für die Zielerreichung geben. Die Konzentration auf Quartiersebene im Bestand reduziert diese Komplexität und bietet die Chance, mehrere Akteure zu vernetzen und zu einer gemeinsamen Durchführung energetischer Maßnahmen zu aktivieren. Hierbei können Skaleneffekte zu höheren $\mathrm{CO}_{2}$-Einsparungen und geringeren finanziellen Belastungen führen. Sowohl im Neubau als auch im Bestand lassen sich zudem effizientere Wärmeversorgungslösungen verwirklichen, die auf Einzelgebäudeebene nicht realisierbar wären. Zusätzliche Themenfelder wie klimagerechte Mobilitätsangebote und Klimaanpassungsmaßnahmen können im Quartier ebenfalls nutzbringender einbezogen werden.

\section{Urbanisierung klimafreundlich gestalten}

Bei der Planung und Realisierung von Neubauquartieren kann die Stadt Hamburg bereits auf ein wirkmächtiges Instrumentarium zugreifen, um Aspekte einer klimafreundlichen Wärmeversorgung sowie einer hohen Gebäudeeffizienz frühzeitig einzubeziehen. Auf Grundlage des Hamburger Klimaschutzgesetzes ( $\$ 4$ HmbKliSchG) werden energetische Festsetzungen in Bebauungsplänen und Regelungen in städtebaulichen Verträgen vorgenommen. Darüber hinaus werden in Grundstückskaufverträgen und Konzeptausschreibungen für Grundstücke energetische Anforderungen an ein Neubauvorhaben gebunden. Im Zuge des Stadtentwicklungsprojekts HafenCity sowie weiterer neuer Quartiersentwicklungsprojekte, die unter Mitwirkung der städtischen Projektentwicklungsgesellschaft IBA realisiert worden sind, konnte dank genannter Instrumente gezeigt werden, dass eine Metropole wie Hamburg klimafreundlich wachsen kann.

Seit 2018 erprobt die Stadt darüber hinaus ein standardisiertes Vorgehen zur Berücksichtigung des Klimaschutzes in Bebauungsplanverfahren. Neubauplanungen mit mehr als 150 Wohneinheiten und einer Geschossflächenzahl von über 
0,8 sollen zukünftig zur regelhaften Erstellung von Energiefachplänen verpflichtet werden. Ab diesen Richtgrößen lässt sich eine leitungsgebundene Wärmeversorgung wirtschaftlich darstellen (MegaWATT 2016). Bezogen auf die wesentlichen Schritte des Bebauungsplanverfahrens fordert die zuständige Behörde in ihrer Rolle als Träger öffentlicher Belange zum Zeitpunkt der Grobabstimmung (Scoping) die Beauftragung und Erstellung eines Energiefachplans. In diesem werden Varianten unterschiedlicher Dämmstandards in Kombination mit klimafreundlichen Wärmeversorgungsvarianten von einem durch den Planungsträger beauftragten Planungsbüro untersucht. Im Ergebnis wird unter Berücksichtigung der örtlichen Rahmenbedingungen für das jeweilige Neubauquartier eine Versorgungslösung identifiziert, die die geringsten $\mathrm{CO}_{2}$-Emissionen bei wirtschaftlicher Vertretbarkeit aufweist. Die daraus resultierenden Anforderungen und Kriterien können über energetische Festsetzungen in Bebauungsplänen oder über Regelungen in städtebaulichen Verträgen festgelegt werden. Mit dem Energiefachplan wird gewährleistet, dass für jede energetische Festsetzung eine fachliche Planung und damit eine sichere Abwägungsgrundlage zugrunde liegen. Fällt die Auswahl auf eine leitungsgebundene Wärmeversorgung, wird ein Anschluss- und Benutzungsgebot festgesetzt. Dadurch wird die Erfüllung dieser Festsetzung zur öffentlichen Aufgabe. Diese zieht eine Ausschreibungspflicht für die Wärmeversorgung des Bebauungsgebiets nach sich, weil die Stadt Hamburg die Rolle eines Energieversorgers nicht selbst übernimmt. Im Rahmen einer öffentlichen Ausschreibung bekommt der Energiedienstleister den Zuschlag für die Wärmelieferkonzession, der die definierten Anforderungen, insbesondere $\mathrm{CO}_{2}$-Einsparungen mit den niedrigsten Wärmepreisen, umsetzen kann.

\section{Transformation des Gebäudebestandes}

Neubauprojekte wirken sich mit der zu erwartenden Flächenentwicklung merklich auf die Klimabilanz Hamburgs aus. Gleichwohl muss der Fokus mindestens ebenso ehrgeizig auf den Gebäudebestand gerichtet sein. In Hamburg wurden über 80 Prozent des Gebäudebestandes vor 1984 errichtet, das heißt vor Einführung der ersten Wärmeschutzverordnung. Folglich bedarf es einer ambitionierten Transformation des Gebäudebestands, um mittels energetischer Gebäudesanierungsmaßnahmen eine maßgebliche Emissionsreduktion zu erzielen. Angesichts der aktuell ausgestalteten energiepolitischen Steuerungsinstrumente setzt die Stadt Hamburg diesen Transformationsprozess mit der Initiierung und Unterstützung von energetischen Quartierskonzepten in Gang. Hauptziel ist die Steigerung der Energieeffizienz unter Berücksichtigung Erneuerbarer Energien sowie konkrete Ansätze für das Auflösen von Gebäudesanierungsstaus. Im Konzept werden somit detaillierte technische und wirtschaftliche Energieeinsparpotenziale im Quartier geprüft, um auf dieser Basis konkrete Maßnahmen für eine kurz-, mittel- und langfristige $\mathrm{CO}_{2}$-Emissionsreduktion zu identifizieren und Umsetzungsschritte vorzuschlagen.
Durch die Inanspruchnahme des Förderprogramms 432 „Energetische Stadtsanierung“ der Kreditanstalt für Wiederaufbau (KfW) wurden in Hamburg seit 2012 acht energetische Quartierskonzepte gefördert. So hat der Bauverein der Elbgemeinden eG auf Basis des erstellten Konzepts ein strommarktorientiertes BHKW in Kombination mit Wärmepumpen und Solarthermie in Betrieb genommen, um die bestehende leitungsgebundene Wärmeversorgung des Quartiers Heidrehmen klimafreundlicher zu gestalten. Eine andere Wohnungsgenossenschaft, der Eisenbahnbauverein eG, hat einen Eisspeicher in Kombination mit einer Gaswärmepumpe errichten lassen. Die HANSA Baugenossenschaft hat sich im Anschluss an die Förderung eines energetischen Quartierskonzepts für die Verlegung eines mit Solarwärme gespeisten Nahwärmenetzes in ihrem Quartier am Dudenweg entschieden. Die Bereitstellung von Landesfördermitteln der Investitions- und Förderbank Hamburg (Programm „Erneuerbare Wärme“) kann solchen Konzepten zur Wirtschaftlichkeit verhelfen.

Grundvoraussetzung für eine erfolgreiche Umsetzung eines energetischen Quartierskonzepts ist die Mitwirkungsbereitschaft der Akteure. Öffentliche Liegenschaften können als Keimzelle eine wichtige Rolle spielen. Gleichzeitig muss Handlungsbedarf für Modernisierungsmaßnahmen vorliegen, dessen Ziele in einer Kooperationsvereinbarung festgelegt werden können. Eine finanzielle Beteiligung der unterzeichnenden Akteure an den Kosten des Quartierskonzepts kann für eine zusätzliche Absicherung der Umsetzungsbereitschaft sorgen. Ferner hat die Vergangenheit gezeigt, dass der Umsetzungserfolg steigt, wenn nur wenige Bestandshalter im Quartier beteiligt werden sowie zusätzliche „Kümmerer“, beispielsweise über das KfW-Programm 432 bezuschusste Sanierungsmanager, für eine effiziente Untersuchung und Umsetzungsbegleitung sorgen. Um energetische Quartierskonzepte in eine breite Umsetzung zu bringen, hat die Stadt Hamburg darüber hinaus im Rahmen der Vereinbarung „Bündnis für das Wohnen“ den wichtigsten Partner, die Wohnungsunternehmen, für das Thema sensibilisiert. Die wohnungswirtschaftlichen Verbände erklären sich darin bereit, auf eine intensive Zusammenarbeit im Quartiersbereich hinzuwirken (BSW 2016).

\section{Aufbau eines Wärmekatasters}

Entscheidend für den Einsatz der aufgeführten Instrumentarien bei der kommunalen Wärmeplanung ist eine umfassende, gesamtstädtische Daten- und Informationsgrundlage der Wärmeversorgungsstruktur. Zu diesem Zweck hat die Stadt Hamburg ein Wärmekataster aufgebaut, welches die Wärmebedarfs-, Wärmeverteilungs- und Energieerzeugungsstrukturen abbildet. Auf einem Online-Portal wird das Wärmekataster der Öffentlichkeit zur Verfügung gestellt, um die nötige Transparenz sowie eine einheitliche Informationsbasis zwischen allen Akteuren zu schaffen.

Das Wärmekataster kann bei der Identifizierung von energetischen Quartieren sowie energieeffizienten Maßnahmen in 
einem bestimmten Gebiet, der Koordination von Infrastrukturmaßnahmen im Energieversorgungsbereich und bei der Verzahnung von Stadtentwicklung und Bauleitplanung sinnvoll unterstützen. Dank des ganzheitlichen Überblicks der örtlichen Gegebenheiten und Potenziale lässt sich mit System Handlungsbedarf erkennen. Insbesondere für den Ausbau und Neubau von leitungsgebundener Wärmeversorgung ist die Kenntnis über bestehende Leitungsstrukturen sowie über Wärmenetzpotenziale ausschlaggebend.

\section{Ausblick}

Das beschriebene Instrumentarium sowie die Vielzahl an Maßnahmen ermöglichen eine zielgerichtete Steuerung der Transformation des Gebäudesektors sowie - zumindest partiell - des gesamten Wärmesektors. Allerdings werden diese allein nicht zur Erreichung der gesetzten Ziele verhelfen. Ein Blick auf Hamburgs Heizungsstatistik verdeutlicht es sehr beispielhaft: Über 70 Prozent der Wohngebäude in Hamburg werden über Zentralheizungen versorgt, wobei mehr als die Hälfte der messpflichtigen Heizungsanlagen älter als 20 Jahre ist (StaNord 2014, LIV 2016). Die Steuerung der zeitnahen Investitionen, die angesichts dieses veralteten Kesselbestands zu erwarten sind, kann nicht ausschließlich mithilfe energetischer Quartierssanierungsprojekte bewältigt werden. Hier gilt es kurzfristig die richtigen Rahmenbedingungen zu setzen, um den Einsatz erneuerbarer Wärme im Gebäudebestand zu befördern und über Jahrzehnte verankerte Fehlinvestitionen in Techniken auf Basis fossiler Energieträger mit sehr langen Investitionszyklen zu vermeiden. Dafür bedarf es einer Stärkung der Wettbewerbsfähigkeit erneuerbarer Energien im Wärmemarkt, beispielsweise über eine gezielte Ausrichtung der Energieträgerkosten am $\mathrm{CO}_{2}$-Gehalt. Zudem ist der Fokus auf die Weiterentwicklung des Regulierungs- und Förderrahmens energetischer Gebäudesanierungsmaßnahmen zu legen, um die seit Jahren auf einem niedrigen Niveau stagnierende Sanierungsrate wirksam anzuheben. Ein Blick auf den Anteil Erneuerbarer Energien an der Wärmeerzeugung, der in Hamburg im Jahr 2018 bei rund vier Prozent lag (BUE 2018), verdeutlicht die immensen Herausforderungen, die es in naher Zukunft zu bewältigen gilt. Dafür bedarf es vor allem der Erarbeitung eines gut austarierten Ordnungsrechts, einer darauf aufbauenden, wirkmächtigen Förderlandschaft sowie ergänzender klimapolitscher Steuerungselemente, bei der die Partizipation wichtiger Akteure Hamburgs geboten ist.

\section{Literatur}

BSW [Behörde für Stadtentwicklung und Wohnen] (2016): Vereinbarung Bündnis für das Wohnen in Hamburg. Im Internet unter:

https://www.hamburg.de/bsw/buendnis-fuer-das-wohnen/.

BUE [Behörde für Umwelt und Energie] (2018): Hamburgs Energie- und Verursacherbilanz, eigene Berechnungen auf Basis der Energieund $\mathrm{CO}_{2}$-Bilanzen für Hamburg des Statistikamt Nord und der Anwendungsbilanzen der AG Energiebilanzen e. V.

Landesinnungsverband des Schornsteinfegerhandwerks Hamburg LIV (2016): Erhebungen des Schornsteinfegerhandwerks für 2016.

MegaWATT Ingenieurgesellschaft mbH (2016): Aktualisierung des Kurzgutachtens über einen Kriterienkatalog für die Festsetzungen nach dem HmbKliSchG im Rahmen des Bebauungsplanverfahrens.

StaNord [Statistikamt Nord] (2014): Zensusergebnisse Gebäude, Wohnung und Haushalte in Hamburg - Informationen im Internet unter: https://www.statistik-nord.de/zahlen-fakten/zensus/zensus/ ergebnisse-fuer-hamburg/.

\section{AUTORIN + KONTAKT}

Lubow Hesse ist Referentin im Amt Energie und Klima der Behörde für Umwelt und Energie in der Freien und Hansestadt Hamburg.

BUE, Neuenfelder Str. 19, 21109 Hamburg. Tel.: +49 40428402732 ,

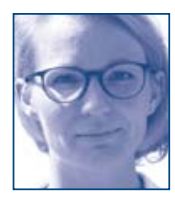

E-Mail: lubow.hesse@bue.hamburg.de

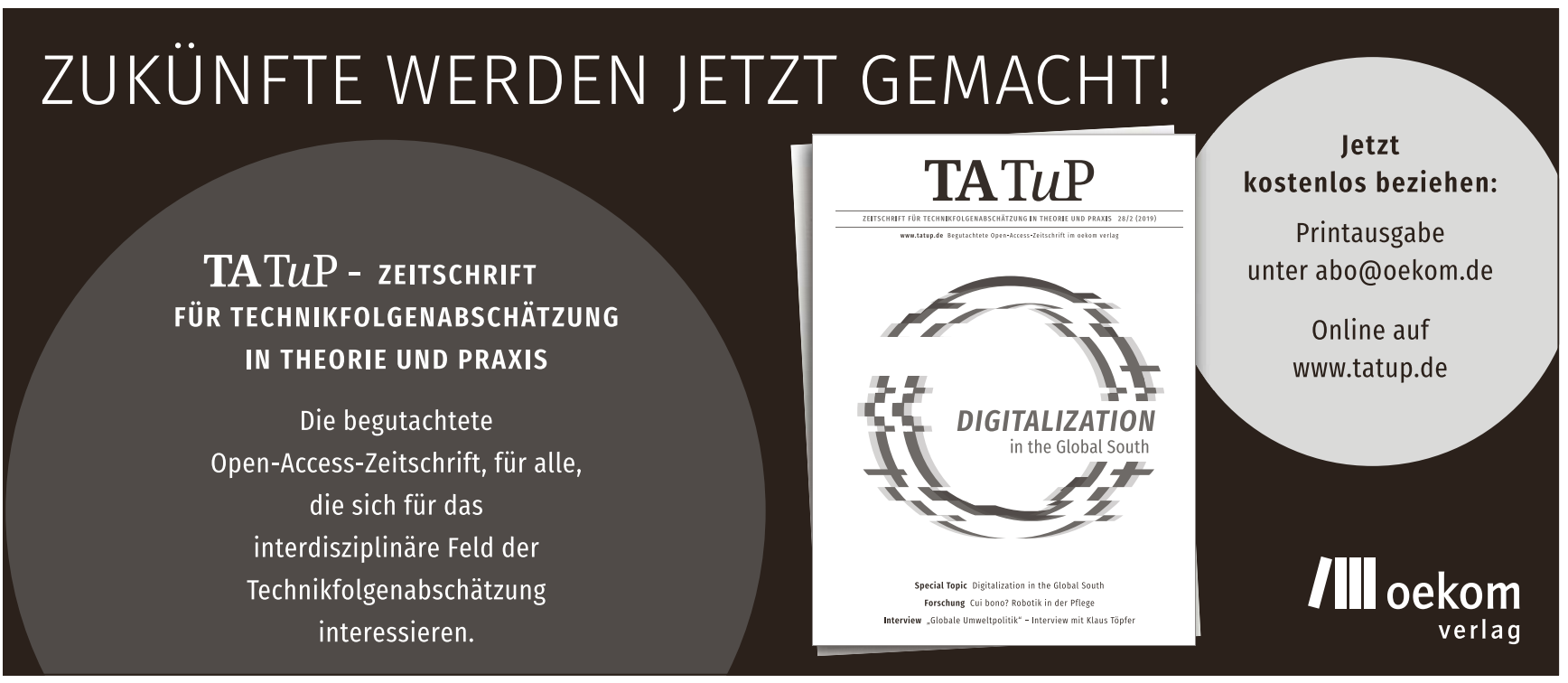

